\title{
The influence of response competition on cerebral asymmetries for processing hierarchical stimuli revealed by ERP recordings
}

\begin{abstract}
It is widely accepted that the left and right hemispheres differ with respect to the processing of global and local aspects of visual stimuli. Recently, behavioural experiments have shown that this processing asymmetry strongly depends on the response competition between the global and local levels of a stimulus. Here we report electrophysiological data that underline this observation. Hemispheric differences for global/ local processing were mainly observed for responseincompatible stimuli and were most prominent between 320 and $400 \mathrm{~ms}$ after stimulus onset. These results underpin the idea that hemispheric differences are more likely to show up when a more elaborated stimulus representation is needed for triggering the response, that is, when a response conflict has to be resolved.
\end{abstract}

Keywords ERP · Global/local processing · Brain asymmetry $\cdot$ Visual attention - Response competition

\section{Introduction}

To interact efficiently in an environment that consists of several interconnected hierarchical levels, it is necessary to process, code and represent objects of different levels in parallel. For instance, while admiring a wonderful buffet, its constituent parts, for example meatballs, chicken wings and lobsters, have to remain accessible, too. Accordingly, a brain mechanism is required that is able to integrate different aspects of a scene while keeping them apart at the same time. A division of labour between the left and right hemisphere is thought to sub-

P. Malinowski ( $)$ T. Gruber

Cognitive Neuroscience and Neuropsychology,

Department of Psychology, University of Liverpool,

Eleanor Rathbone Building, Liverpool L69 7ZA, UK

e-mail: petermal@liverpool.ac.uk

Tel.: +44-151-7946706, Fax: +44-151-7946937

R. Hübner · A. Keil

Fachgruppe Psychologie, Universität Konstanz, Konstanz, Germany serve this goal, with the right hemisphere $(\mathrm{RH})$ being specialised for processing global, overall aspects of a stimulus (the buffet), and the left hemisphere (LH) for processing its local parts (the lobsters).

Although neuropsychological, electrophysiological, brain imaging and behavioural studies yield ample evidence in support of this idea, the underlying brain processes are still under dispute. In particular, it is discussed as to whether attentional or sensory processes play the major role (see, for example, Hübner 1997, 1998; Ivry and Robertson 1998; Robertson and Lamb 1991). This question was addressed directly in several behavioural studies by contrasting conditions with and without response competition between the global and local level (Hübner 1997; Hübner and Malinowski 2002). A central cue preceded each laterally presented hierarchical stimulus (large global letters constructed by arranging several identical local letters), and indicated which level was relevant on a trial-by-trial basis. Since the task was to classify which of four letters, mapped to two response categories, appeared on the cued level the relation between the two levels was response compatible, when global and local letters led to the same response, or response incompatible, when they required different responses. One major finding was that response times (RTs) for the global level were slower for right visual field stimuli (LH processing) than for left visual field stimuli, mainly when global and local levels were incompatible. Local responses were slower when the stimulus appeared in the left visual field (RH processing), compared to when it appeared in the right visual field, again mainly when the levels were incompatible. The fact that response competition was the key factor in producing these asymmetric visual field effects suggests that higher level processes, presumably attentional processes, and not only sensory mechanisms contribute to them. As we (Hübner and Malinowski 2002) proposed, the corresponding brain asymmetries might only become prominent when a more elaborated representation of the stimulus is needed to solve the task. Early and incomplete representations of the global and local level might, 

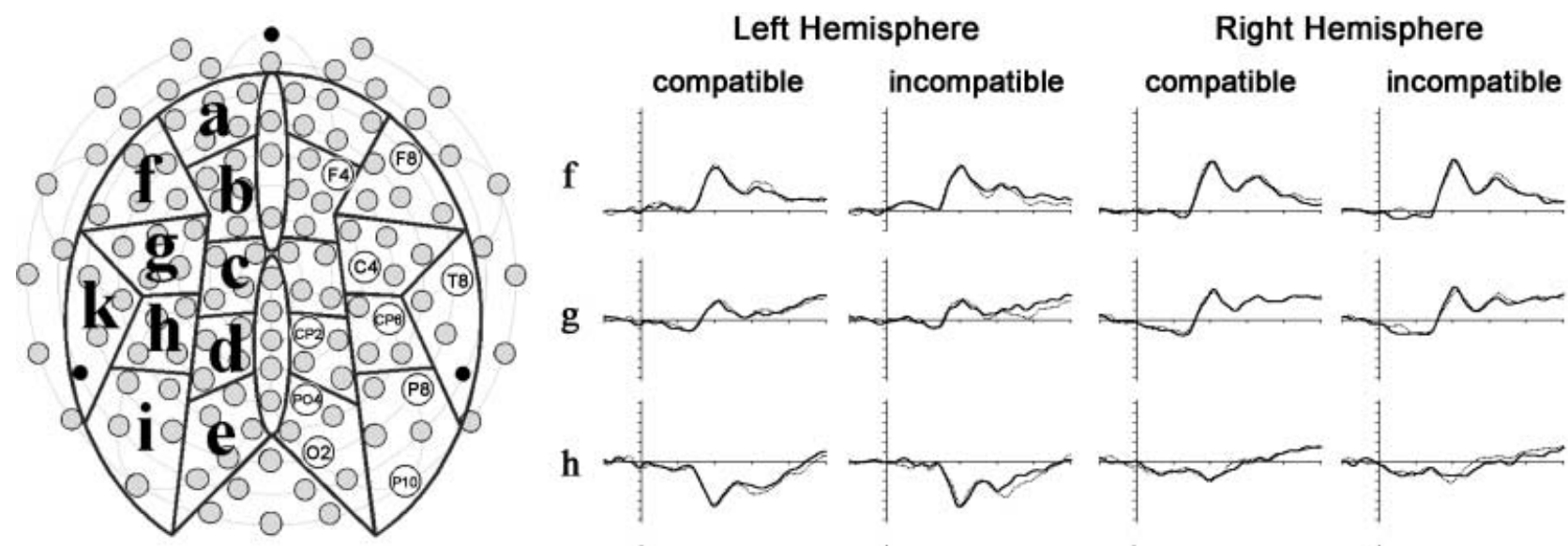

Right Hemisphere
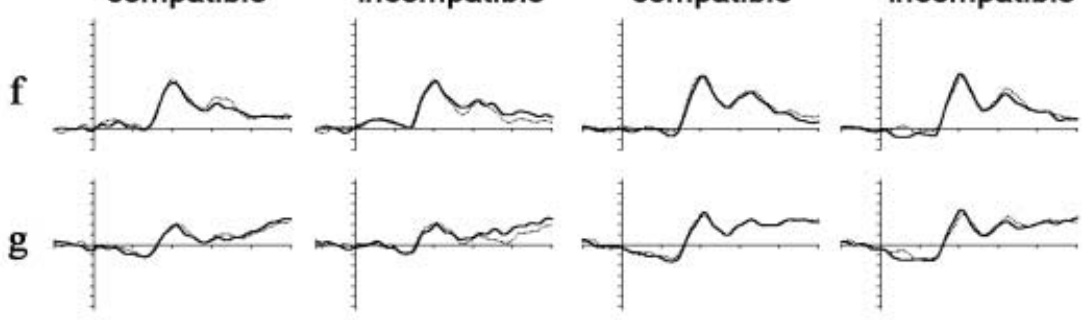

h
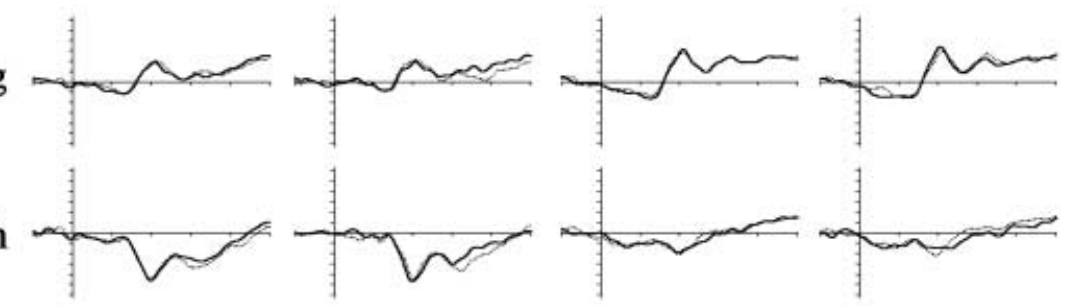

d
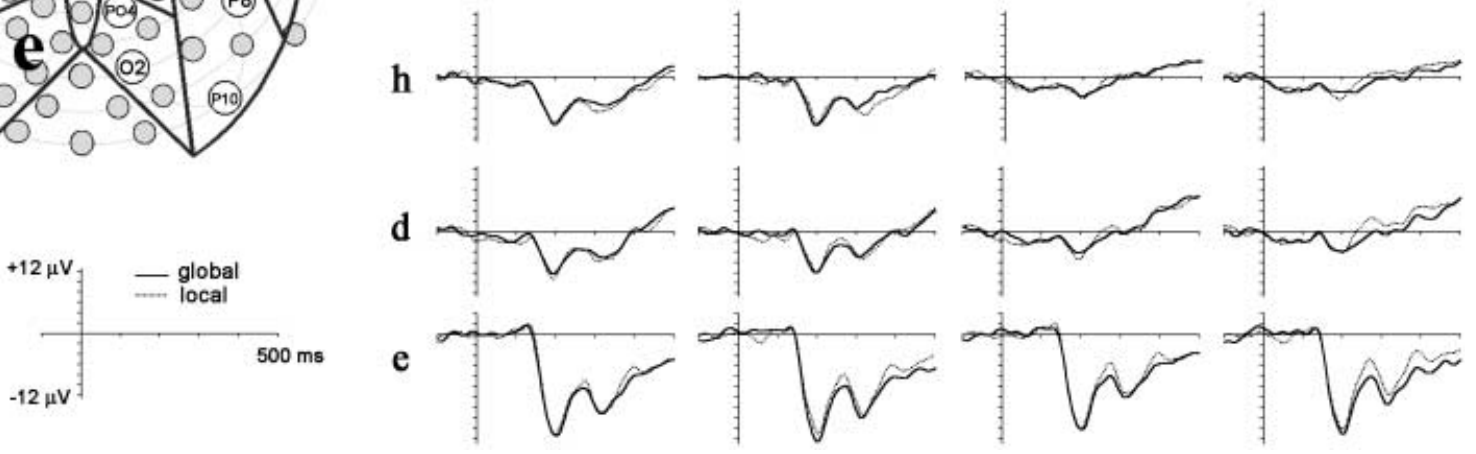

Fig. 1 Left Arrangement of the 128-channel electrode array and the locations of the grouped electrodes $(a-i, k)$ of the left and right hemispheres. For orientation some electrodes of the right hemisphere are labelled according to the extended 10-20 system. Right Regional mean event-related potentials across the 11 subjects for five out of ten electrode groups over each hemisphere $(f, g, h, d, e)$. Stimulus onset corresponds to time point $0 \mathrm{~ms}$

on the other hand, automatically activate the related responses, while a later and complete representation is only used when a response conflict occurs.

In the present study we recorded event-related potentials (ERPs), to test whether our findings from behavioural experiments can be confirmed with psychophysiological measures. If the above-mentioned hypothesis holds, global/local ERP amplitudes and topographies over the LH and RH should be similar when stimuli are response compatible, while they should differ for incompatible stimuli, because in the latter case a more elaborated stimulus representation is used to solve the task.

To match our behavioural studies as closely as possible we used a very similar experimental procedure, with the main difference being that stimuli were now presented centrally on the screen. To our knowledge, only one other ERP study examined the relationship between hemispheric asymmetries in a global/local task and the allocation of attention by means of a cuing paradigm (Yamaguchi et al. 2000). While in that study a detection paradigm was employed, here for the first time we report electrophysiological data with a classification task and randomised target levels.

\section{Materials and methods}

Sixteen compound stimuli were created by combining four different letters, where global letters (visual angle: $3.3^{\circ}$ horizontally, $4.5^{\circ}$ vertically) were constructed from several identical local

letters (visual angle: $0.5 \times 0.7^{\circ}$ ) arranged within a $5 \times 5$ grid. Each local letter was constructed from white lines describing the outline of the respective letter. The stimuli appeared on a black background (for details see Hübner and Malinowski 2002).

Fourteen right-handed participants (seven male, seven female, mean age 24.1 years) took part in the experiment after giving informed consent. Three of them had to be excluded due to excessive artefacts. Their task was to classify the letter on the relevant level by pressing one out of two response buttons with their index or middle finger of the same hand ("A" and "S" mapped to one button; " $\mathrm{H}$ " and "E" to the other). Mapping of the letter pairs to the response keys and responding hand were counterbalanced across subjects. Each trial started with a fixation cross and a cue for $300 \mathrm{~ms}$. The cue was the outline of a large or small rectangle, the size corresponding to that of the relevant level. After a blank screen of $340 \mathrm{~ms}$ the stimulus was presented for $100 \mathrm{~ms}$. The intertrial interval varied randomly between 1,100 and $1,600 \mathrm{~ms}$. The two factors, LEVEL (global, local) and RESPONSE COMPATIBILITY (compatible, incompatible) were randomised within each block of trials. After one practice block, six blocks of 96 trials each were run.

An EEG was recorded continuously with an EGI (Electrical Geodesics, 1998) 128-electrode array (impedances <50 k $\Omega$; sampling rate $250 \mathrm{~Hz} ; 0.1-200 \mathrm{~Hz}$ online bandpass filter, referenced to $\mathrm{Cz}$ ) and segmented to obtain epochs containing $300 \mathrm{~ms}$ prior to cue onset and $800 \mathrm{~ms}$ following stimulus onset. Artefact correction was performed by means of the 'statistical correction of artefacts in dense array studies' (SCADS) procedure (Junghöfer et al. 2000). For further analysis the average reference was used and a 30-Hz lowpass filter was applied.

For each hemisphere the electrodes were separated into ten groups $(\mathrm{a}-\mathrm{i}, \mathrm{k})$ consisting of either four or five neighbouring electrodes (see Fig. 1), similar to the subdivision used by Yamaguchi et al. (2000). For the statistical analyses, ERP amplitudes within each electrode group were normalised (McCarthy and Wood 1985) and averaged. They were referred to a prestimulus baseline of 100 to $0 \mathrm{~ms}$ before stimulus onset.

\section{Results and discussion}

ANOVAs of the RTs and error rates showed that responses to incompatible stimuli were slower $[F(1,10)=$ $29.44, P<0.001]$ and produced more errors $[F(1,10)=$ 
Table 1 Mean response times ( $R T$; in milliseconds) and error rates (in percent)

\begin{tabular}{lccccc}
\hline & \multicolumn{2}{l}{ Compatible } & & \multicolumn{2}{l}{ Incompatible } \\
\cline { 2 - 3 } \cline { 6 - 6 } \cline { 5 - 6 } & Global & Local & & Global & Local \\
\hline RT means & 574 & 599 & & 619 & 621 \\
Error means & 2.7 & 3.2 & & 6.6 & 6.5 \\
\hline
\end{tabular}

14.20, $P<0.005]$. For RTs the interaction between both factors was marginally significant $[F(1,10)=3.38$, $P=0.096]$. No other effects were significant. The mean RTs and error rates are given in Table 1 . The tendency of a stronger interference effect from local on global contradicts early studies in global/local processing, that usually found stronger interference from global on local than vice versa (see, for example, Navon 1977). However, as recently summarised by Shedden and Reid (2001) the global interference effect and the global RT advantage can be altered and even reversed by manipulating perceptual and post-perceptual factors. In the context of the current experiment the RTs and error rates reflect that neither the global nor the local level enjoyed a pronounced processing advantage and that the letterclassification task could easily be solved.

For each scalp site and each analysed time window the mean ERP amplitudes were evaluated statistically using an ANOVA for repeated measures with LEVEL (global vs local), HEMISPHERE (left vs right) and RESPONSE COMPATIBILITY (compatible vs incompatible) as independent factors.

The expected asymmetric ERP pattern for incompatible stimuli was most prominent in the interval between 320 and $400 \mathrm{~ms}$, and was statistically expressed as an interaction between LEVEL, HEMISPHERE and RESPONSE COMPATIBILITY. This effect was significant at central, parietal and temporal sites [site c: $F(1,10)=13.89, \quad P<0.005 ; \quad$ site d: $\quad F(1,10)=91.43$, $P<0.0001 ;$ site h: $F(1,10)=8.60, \quad P<0.05 ;$ site k: $F(1,10)=8.74, P<0.05$; site g: $F(1,10)=9.15, P<0.05]$. It reflects the fact that mainly for incompatible stimuli there was a hemispheric asymmetry for global/local processing. Over the LH the amplitudes were more negative for local/incompatible stimuli, while over the RH the amplitudes were more negative for global/incompatible stimuli (see Fig. 1). This is also confirmed by scalp topographies of the difference waves between global and local processing during this interval. As Fig. 2 shows, they have a largely symmetrical topography for compatible conditions. For incompatible conditions the difference is positive over temporal parietal and temporal occipital areas of the LH. Note that in this interval amplitudes were negative, thus positive values reflect larger negativity for the local condition. Over occipital parietal areas of the RH the difference is negative, thus reflecting larger negativity for the global condition.

While the general pattern of asymmetric activation fits well with previous ERP studies on global/local brain

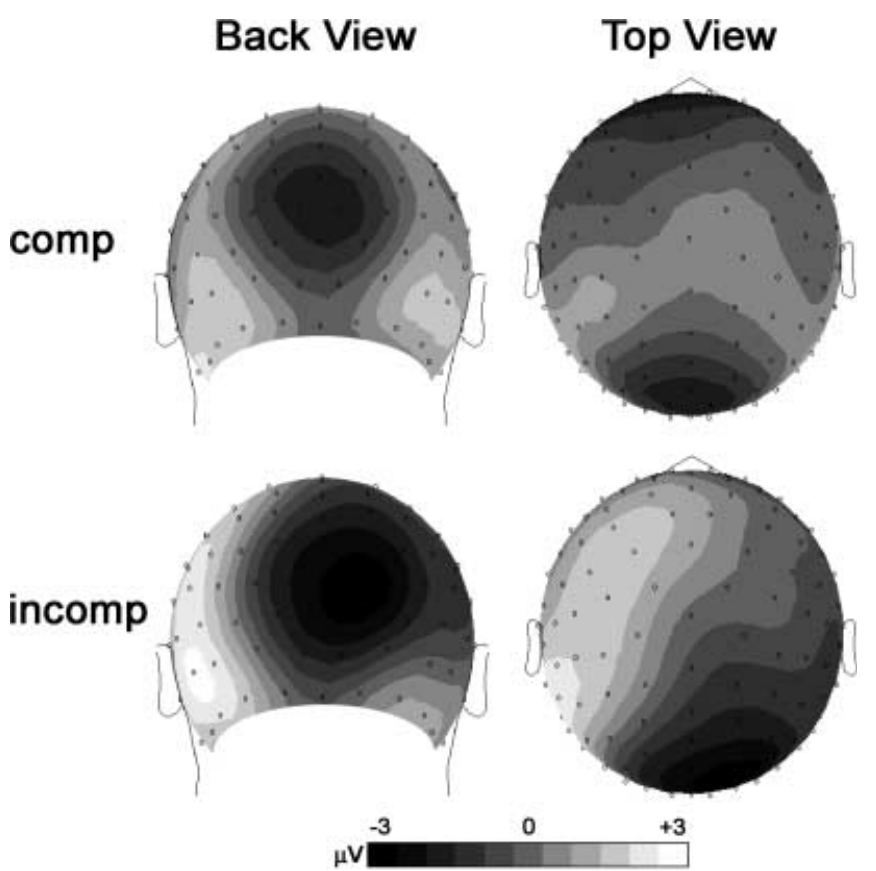

Fig. 2 Spherical spline interpolated topographies of the grand mean difference waves (global minus local), averaged over the interval between 320 and $400 \mathrm{~ms}$, for compatible (comp) and incompatible (incomp) conditions

asymmetries, the evoked responses were slightly delayed in our study. For instance, in a study by Proverbio et al. (1998) asymmetries occurred between 200 and $400 \mathrm{~ms}$ after stimulus onset. In studies by Heinze et al. (1998) and Yamaguchi et al. (2000) these asymmetries started around $260 \mathrm{~ms}$. Since in these three studies a detection task was employed, the delay in our study might be explained by higher processing demands due to the classification task. Interestingly, in the study by Heinze et al. (1998) these asymmetries only occurred when the task was to attend to the local and global level simultaneously (divided attention). For local target letters a stronger negativity over left posterior areas was observed. For global targets, however, this negativity was more pronounced over right posterior areas. In contrast, no such asymmetry emerged when within one experimental block either the global or the local level was relevant. Note, that for defining global and local targets in a divided attention task, it is necessary that the global and local levels are always different (incongruent). In accord with these results, in all brain imaging studies that reported asymmetric cerebral activation, the global/local stimuli were always incongruent, too (Fink et al. 1996, 1997, 1999; Martinez et al. 1997). On the other hand, Proverbio et al. (1998) reported asymmetries also for focused attention and congruent stimuli. Evans et al. (2000) showed that the asymmetric ERP patterns depend on the identity of the irrelevant level. When it contained invariable and neutral elements global/local processing asymmetries appeared already in the P1 component peaking around $100 \mathrm{~ms}$. In contrast, variable, task-rele- 
vant information on the irrelevant level resulted in processing asymmetries that occurred in the time range of 200-400 ms.

The results of the present study support the idea that cerebral asymmetries for global/local processing depend on how elaborated the stimulus representation is that is needed to solve the task. When a fast and incomplete representation suffices, no appreciable asymmetries occur. This was the case when the global and local levels were response compatible. However, when an elaborated representation is needed because a target can occur at both levels, as in Heinze et al. (1998), or because a response conflict occurred, as in the present study, a processing asymmetry becomes obvious. On the other hand, with very low processing demands asymmetries might also occur for compatible stimuli (Proverbio et al. 1998). When the irrelevant level remains unchanged throughout trials they might even show up in the P1 component (Evans et al. 2000).

As Fig. 2 shows this left-right asymmetry itself is asymmetrical. While the focus of stronger local activity is largely lateralised to the left and is located more temporally, the focus of stronger global activity is closer to the midline and has a more parietal location. It is conceivable that more spatial information is needed to recognise global letters since they are defined by the spatial arrangement of the local letters. Thus the dorsal stream, carrying the largest amount of spatial information (Ungerleider and Haxby 1994), might be more involved in the global than in the local task, where less spatial information is needed. However, future research has to show whether this assumption holds or other factors contribute to the differential topography over the LH and RH.

To sum up, the present data yield support for the hypothesis that the completeness of stimulus representation might be an important factor in determining global/local brain asymmetries and might also explain some of the variability of the data in this field. The type of task and the demands posed by it might have to be taken into account as well.

Acknowledgements We would like to thank Ulla Martens and Ingeborg Schweiss for their help in data recording and analysis and Nicola Williams for editorial assistance. This work was supported by a grant from the German Research Foundation (DFG, Ro 805/11-1).

\section{References}

Evans MA, Shedden JM, Hevenor SJ, Hahn MC (2000) The effect of variability of unattended information on global and local processing: evidence for lateralization at early stages of processing. Neuropsychologia 38:225-239

Fink GR, Halligan PW, Marshall JC, Frith CD, Frackowiak RSJ, Dolan RJ (1996) Where in the brain does visual attention select the forest and the trees? Nature 382:626-628

Fink GR, Halligan PW, Marshall JC, Frith CD, Frackowiak RSJ, Dolan RJ (1997) Neural mechanisms involved in the processing of global and local aspects of hierarchically organized visual stimuli. Brain 120:1779-1791

Fink GR, Marshall JC, Halligan PW, Dolan RJ (1999) Hemispheric asymmetries in global/local processing are modulated by perceptual salience. Neuropsychologia 37:31-40

Heinze HJ, Hinrichs H, Scholz M, Burchert W, Mangun GR (1998) Neural mechanisms of global and local processing: a combined PET and ERP study. J Cogn Neurosci 10:485-498

Hübner R (1997) The effect of spatial frequency on global precedence and hemispheric differences. Percept Psychophys 59:187-201

Hübner R (1998) Hemispheric differences in global/local processing revealed by same-different judgements. Vis Cogn 5:457478

Hübner R, Malinowski P (2002) The effect of response competition on functional hemispheric asymmetries for global/local processing. Percept Psychophys (in revision)

Ivry RB, Robertson LC (1998) The two sides of perception. MIT Press, Cambridge, MA

Junghöfer M, Elbert T, Tucker DM, Rockstroh B (2000) Statistical control of artifacts in dense array EEG/MEG studies. Psychophysiology 37:523-532

Martinez A, Moses P, Frank L, Buxton R, Wong EC, Stiles J (1997) Hemispheric asymmetries in global and local processing: evidence from fMRI. Neuroreport 8:1685-1689

McCarthy G, Wood CC (1985) Scalp distributions of event-related potentials: an ambiguity associated with analysis of variance models. Electroencephalogr Clin Neurophysiol 62:203-208

Navon D (1977) Forest before the trees: the precedence of global features in visual perception. Cogn Psychol 9:353-393

Proverbio AM, Minniti A, Zani A (1998) Electrophysiological evidence of a perceptual precedence of global vs. local visual information. Cogn Brain Res 6:321-334

Robertson LC, Lamb MR (1991) Neuropsychological contributions to theories of part/whole organization. Cogn Psychol 23:299-330

Shedden JM, Reid GS (2001) A variable mapping task produces symmetrical interference between global information and local information. Percept Psychophys 63:241-252

Ungerleider LG, Haxby JV (1994) "What" and "where" in the human brain. Curr Opin Neurobiol 4:157-165

Yamaguchi S, Yamagata S, Kobayashi S (2000) Cerebral asymmetry of the 'top-down' allocation of attention to global and local features. J Neurosci 20:1-5 